\title{
Impact of short-incubation MALDI-TOF MS on empiric antibiotic therapy in bloodstream infections caused by Pseudomonas aeruginosa, Enterococcus spp. and AmpC-producing Enterobacteriaceae
}

\author{
Mika Halavaara ${ }^{\mathrm{a}, *}$, Annika Nevalainen ${ }^{\text {b }}$, Timi Martelius ${ }^{\text {a }}$, Pentti Kuusela ${ }^{\text {b }}$, Veli-Jukka Anttila ${ }^{a}$ \\ a Department of Infectious Diseases, Inflammation Center, Helsinki University Hospital and University of Helsinki, Finland \\ b Division of Clinical Microbiology, HUSLAB, Helsinki University Hospital and University of Helsinki, Finland
}

\section{A R T I C L E I N F O}

Article history:

Received 6 September 2018

Received in revised form 1 November 2018

Accepted 18 November 2018

Available online $\mathrm{xxxx}$

\section{Keywords:}

MALDI-TOF

Short incubation

Bacteremia

Bloodstream infection

Empiric antibiotic treatment

\begin{abstract}
A B S T R A C T
The impact of the short-incubation matrix-assisted laser desorption ionization time-of-flight (si-MALDI-TOF) mass spectrometry technique was evaluated in the treatment of bloodstream infections (BSIs) caused by Pseudomonas aeruginosa, Enterococcus spp., and Amp-C producing Enterobacteriaceae. A total of 124 bacteremia episodes were divided into 2 groups: i) si-MALDI-TOF group $(n=69)$ and ii) control group $(n=55)$. Identification by si-MALDI-TOF resulted in 12.8\% increase in cases receiving appropriate antibiotic treatment within $48 \mathrm{~h}$ from blood culture draw. The importance of the rapid identification was emphasized in BSIs caused by enterococci $(n=62$ ), where si-MALDI-TOF led to appropriate antibiotic treatment in $87.9 \%$ of cases (versus control group $65.5 \%, P=0.036$ ). Implementation of si-MALDI-TOF technology for microbial identification was associated with increased proportion of patients receiving effective antibiotic treatment within $48 \mathrm{~h}$ from blood culture draw. The effect was most significant in BSIs caused by enterococcal species and in a subgroup of immunosuppressed patients.
\end{abstract}

(c) 2018 Elsevier Inc. All rights reserved.

\section{Introduction}

The cornerstone of the management of bloodstream infections (BSIs) is rapid identification of the causative organism (Seifert, 2009). This will enable timely and appropriate antibiotic treatment, which is the most important predictor of a better outcome in patients with BSI (Ibrahim et al., 2000; Leibovici et al., 1998). This issue is relevant because BSIs cause a significant burden of disease with an increasing incidence (Skogberg et al., 2012).

Matrix-assisted laser desorption ionization time-of-flight (MALDITOF) mass spectrometry has changed the field of rapid microbial identification in clinical microbiology, especially in bacteremias (Drancourt, 2010). This technique allows a shorter time from blood culture draw to organism identification in patients with BSI compared with traditional methods (Bizzini and Greub, 2010; Martiny et al., 2013). Several studies have shown this technique's benefit on antibiotic treatment in patients with BSI (Clerc et al., 2013; Huang et al., 2013; Vlek et al., 2012). Combined with antimicrobial stewardship team intervention, it has been associated with reduced mortality and shorter hospital stay in patients with BSI (Huang et al., 2013). In addition, combined with antimicrobial stewardship team intervention, it has been shown to be cost-effective (Patel et al., 2016; Perez et al., 2013). MALDI-TOF analysis

\footnotetext{
* Corresponding author. Tel.: +358-504270202.

E-mail address: mika.halavaara@hus.fi (M. Halavaara).
}

combined with a short subculture time of positive blood cultures (siMALDI-TOF) is an improvement of this technique, allowing even shorter time to pathogen identification with a positive effect on antibiotic therapy (Idelevich et al., 2014; Kohlmann et al., 2015), and is also associated with reduced length of hospital stay and mortality in patients with BSI (Delport et al., 2017).

In BSIs caused by Pseudomonas aeruginosa, Enterococcus spp., and AmpC-producing Enterobacteriaceae, empiric antibiotic therapy is likely to be inappropriate due to their natural resistance profile. This stresses the need for a faster recognition of these pathogens in bloodstream, especially since the benefit of timely and appropriate antibiotic treatment has been demonstrated also with these pathogens (Huang et al., 2013; Kang et al., 2003; Zasowski et al., 2016). An increase in mortality was observed in patients with delay in receiving appropriate antibiotic therapy over $48 \mathrm{~h}$ from blood culture draw (Lodise et al., 2007; Zasowski et al., 2016). Previous studies have also suggested particular benefit of MALDI-TOF in bacteremias caused by these pathogens (Clerc et al., 2013; Osthoff et al., 2016). However, in most studies, the impact of MALDI-TOF analysis is evaluated in combination with antimicrobial stewardship team and/or infectious disease (ID) consultation.

In the present study, we investigate the impact of si-MALDI-TOF on antibiotic treatment of BSIs caused by Pseudomonas aeruginosa, Enterococcus spp., and AmpC-producing Enterobacteriaceae. We hypothesized that si-MALDI-TOF would enable patients to receive appropriate antibiotic treatment faster. Time from blood culture draw to the receipt of 
appropriate antibiotic therapy is a good indicator of the possible benefits of si-MALDI-TOF (Timbrook et al., 2017). Therefore, the primary outcome of the study was the percentage of patients receiving appropriate antibiotic therapy within $48 \mathrm{~h}$ after the blood culture draw.

\section{Materials and methods}

\subsection{Patients and study design}

This was a retrospective, observational study of adult patients with BSIs caused by Pseudomonas aeruginosa, Enterococcus spp., and AmpCproducing Enterobacteriaceae (including Enterobacter spp., Serratia spp., and Morganella morganii) between March 2014 and December 2015 at Helsinki University Hospital (HUH). Patients were identified from the clinical microbiology laboratory database at Helsinki University Hospital Laboratory (HUSLAB). Only the first BSI episode of patients with multiple episodes of the same bacteria during the study period was included.

HUH is a tertiary hospital serving population about one and a half million in the Hospital District of Helsinki and Uusimaa. The prevalence of multidrug-resistant Pseudomonas aeruginosa is low (65 cases year 2015). Also, the prevalence of vancomycin-resistant enterococci (VRE) is low (under 20 cases annually past few years). The research board of the Inflammation Center at the Helsinki University Hospital approved the study protocol.

\subsection{Identification of bacteria}

The si-MALDI-TOF identification was done from all blood culture bottles (BacT/Alert FA Plus, FN/PF Plus, FA, SN, and PF; bioMérieux) which flagged positive between 8 p.m. and 11 a.m. (si-MALDI-TOF group). For the analysis, 50-100 $\mu \mathrm{l}$ of blood culture content was applied on chocolate agar plates, which were incubated for $3-4 \mathrm{~h}$ at $+35^{\circ} \mathrm{C}$ in $\mathrm{CO}_{2}$. After incubation, a sample for the MALDI-TOF analysis was taken by streaking the application area on the chocolate agar. One spot was inoculated for each sample. The analysis (Vitek MS; bioMérioux) was performed even if no growth was visible. Simultaneously with the siMALDI-TOF analysis, another sample from blood culture bottle was Gram stained and subcultured on agar plates for susceptibility and identification confirmatory testing. Additionally, a sample from a positive blood culture bottle was used for conventional bacterial identification (in-house identification set containing 10 biochemical tests, as well as chromogenic plates). On the next day, a conventional MALDI-TOF was performed in cases giving unreliable identification in conventional biochemical analysis. The si-MALDI-TOF results were reported between 11 a.m. and 2 p.m., and the results for the control group were reported on the next day between 9 a.m. and 11 a.m. As a consequence, identification by si-MALDI-TOF resulted in initial identification roughly 22$24 \mathrm{~h}$ earlier than the one achieved by conventional MALDI-TOF and biochemical identification methods.

\subsection{Data collection and statistics}

An infectious disease specialist (M.H.) reviewed medical records of each patient. Demographic and clinical data were collected. Demographic data were analyzed using descriptive statistics. Categorical variables were analyzed using Chi-square analysis or Fisher's exact test when appropriate. Mann-Whitney $U$ test was used in continuous variables if data were not normally distributed; otherwise, a 2-tailed Student $t$ test was used. Kaplan-Meier method and log-rank test were used to analyze survival (type) data. $P$ value $<0.05$ was considered statistically significant. Statistical analyses were performed using SPSS software, version 22.0 (SPSS, Inc., Chicago, IL).

\subsection{Study definitions}

Index bacteria mean one of the bacteria that are included in the study. Index bacteria are Pseudomonas aeruginosa, Enterococcus spp., and AmpC-producing Enterobacteriaceae (Enterobacter spp., Serratia spp., and Morganella morganii). Polymicrobial bacteremia was defined as the isolation of more than 1 bacterial species during the same bacteremia episode, except when the second microorganism was considered to be a contaminant. The focus or source of BSI was assessed on the basis of medical records.

Immunosuppressive treatment included antirejection medication, cancer chemotherapy, prednisolone more than $10 \mathrm{mg} / \mathrm{day}$, and antirheumatic medications including biological medications (Table 1). Patients in this group are similar in a way that they all receive or have received immunosuppressive medication and are in a close follow-up of appropriate specialty. So, in their case, immunosuppression is clearly defined and is due to medication and comorbidity also. Patients with a disease associated with varying degree of immunosuppression (e.g., diabetes or liver disease) are a more heterogeneous group and harder to define. Thus, patients with these comorbidities are not included in the subgroup analysis including immunosuppressed patients.

\subsection{Study definitions about antibiotic therapy}

Appropriate antibiotic therapy was defined as an antibiotic regimen (i) to which the index bacterium was susceptible in vitro, (ii) considered standard treatment according to Helsinki University Hospital's antimicrobial treatment guide, and (iii) delivered intravenously (with the exception of fluoroquinolones). For example, antipseudomonal beta-lactam or fluoroquinolone for Pseudomonas aeruginosa BSI was considered appropriate, while monotherapy with aminoglycosides was inappropriate. Similarly, first-, second-, and third-generation cephalosporins and monotherapy with aminoglycosides were considered inappropriate for AmpC-producing Enterobacteriaceae. Time to the appropriate antibiotic

Table 1

Patient characteristics and focus or source of infection.

\begin{tabular}{|c|c|c|c|c|}
\hline & $\begin{array}{l}\text { Whole study } \\
\text { group } \\
(n=124)\end{array}$ & $\begin{array}{l}\text { Si-MALDI-TOF } \\
\text { Group } \\
(n=69)\end{array}$ & $\begin{array}{l}\text { Control } \\
\text { group } \\
(n=55)\end{array}$ & $\begin{array}{l}P \\
\text { value }\end{array}$ \\
\hline \multicolumn{5}{|l|}{ Patient characteristics } \\
\hline Age, years (mean; SD) & $61.3 ; 15.2$ & $62.7 ; 15.6$ & $59.6 ; 14.7$ & 0.257 \\
\hline Gender, male; $n(\%)$ & $74(59.7)$ & $42(60.9)$ & $32(58.2)$ & 0.762 \\
\hline Chronic heart disease & $14(11.3)$ & $9(13.0)$ & $5(9.1)$ & 0.490 \\
\hline Chronic kidney disease & $22(17.7)$ & $11(15.9)$ & $11(20.0)$ & 0.557 \\
\hline Dialysis & $4(3.2)$ & $2(2.9)$ & $2(3.6)$ & $>0.99$ \\
\hline Liver cirrhosis & $5(4.0)$ & $4(5.8)$ & $1(1.8)$ & 0.381 \\
\hline Chronic lung disease & $18(14.5)$ & $11(15.9)$ & $7(12.7)$ & 0.614 \\
\hline Diabetes & $26(21.0)$ & $14(20.3)$ & $12(21.8)$ & 0.835 \\
\hline Blood malignancy $^{\mathrm{a}}$ & $14(11.3)$ & $10(14.5)$ & $4(7.3)$ & 0.207 \\
\hline Acute leukemia & $15(12.1)$ & $9(13.0)$ & $6(10.9)$ & 0.717 \\
\hline Stem cell transplant & $5(4.0)$ & $2(2.9)$ & $3(5.5)$ & 0.654 \\
\hline Solid organ malignancy & $32(25.8)$ & $16(23.2)$ & $16(29.1)$ & 0.456 \\
\hline Solid organ transplant & $18(14.5)$ & $10(14.5)$ & $8(14.5)$ & 0.993 \\
\hline $\begin{array}{l}\text { Immunosuppressive } \\
\text { treatment }^{\mathrm{b}}\end{array}$ & $70(56.5)$ & $44(63.8)$ & $26(47.3)$ & 0.066 \\
\hline Neutropenia $^{c}$ & $21(16.9)$ & $14(20.3)$ & $7(12.7)$ & 0.265 \\
\hline \multicolumn{5}{|l|}{ Infection focus or source } \\
\hline Pulmonary & $5(4.0)$ & $2(2.9)$ & $3(5.5)$ & 0.654 \\
\hline Urinary tract & $21(16.9)$ & $13(18.8)$ & $8(14.5)$ & 0.526 \\
\hline Skin/musculoskeletal & $7(5.6)$ & $5(7.2)$ & $2(3.6)$ & 0.461 \\
\hline Abdominal cavity & $55(44.4)$ & $28(40.6)$ & $27(49.1)$ & 0.343 \\
\hline Central nervous system & $1(0.8)$ & $1(1.4)$ & 0 & $>0.99$ \\
\hline Heart & $2(1.6)$ & $1(1.4)$ & $1(1.8)$ & $>0.99$ \\
\hline Indwelling catheter & $5(4.0)$ & $1(1.4)$ & $4(7.3)$ & 0.170 \\
\hline Unknown & $28(22.6)$ & $18(26.1)$ & $10(18.2)$ & 0.296 \\
\hline
\end{tabular}

a Other than acute leukemia.

b Antirejection medication, cancer chemotherapy, prednisolone more than $10 \mathrm{mg} / \mathrm{day}$, and antirheumatic medications including biological medications.

Absolute neutrophil cell count less than $0.5 \times 10^{5}$ neutrophils $/ \mathrm{mL}$. 
therapy was calculated from the time of the first positive blood culture draw to the delivery of first dose of appropriate antibiotic.

\subsection{Exclusion criteria}

Patient exclusion criteria were i) transfer to another hospital before receipt of appropriate antibiotic therapy or lack of verified clinical details (data could not be obtained) $(n=25)$, ii) patient did not receive appropriate antibiotic treatment at all by the decision of the treating physician (e.g., due to poor prognosis) $(n=6)$, iii) bacteremia episodes during the appropriate antibiotic treatment $(n=5)$, and iv) blood culture result ruled as a contamination by the treating physician $(n=4$; in these cases, blood culture was drawn through a catheter and did not correlate with clinical situation).

\section{Results}

\subsection{General}

A total of 164 episodes of bacteremia caused by the index bacteria were identified. According to exclusion criteria, 40 episodes were excluded (as shown in more detail is Subsection 2.6.), and resulting 124 bacteremia episodes originating from 120 patients were included in the study. One patient had 2 separate bacteremias of different index bacteria during the study period. Three patients had a polymicrobial bacteremia caused by 2 of the index bacteria. Consequently, both these findings were analyzed as separate episodes. The number of polymicrobial bacteremias including only 1 of index bacteria was 15 .

One hundred twenty-four bacteremia episodes were divided into 2 groups: i) si-MALDI-TOF group $(n=69)$, i.e., si-MALDI-TOF was used for bacterial identification with a positive result, and ii) control group $(n=55)$, i.e., identification was done by means of conventional methods (including those cases where si-MALDI-TOF was done but was negative). For more details, see Subsection 2.2.

The site of acquisition of the blood culture was emergency room in $33.9 \%(n=42)$, intensive care unit in $12.9 \%$ (16), internal medicine (including pulmonology and neurology) ward in 4.8\% (6), hematology ward in $13.7 \%$ (17), surgical ward in $25.8 \%$ (32), gynecology ward in $3.2 \%$ (4), and oncology ward in 5.6\% (7) of cases.

Baseline demographic characteristics were similar in both study groups (Table 1). HUH is the only national center for solid organ transplantations and also responsible for the majority of stem cell transplantations nationally. This explains the quite high number of hematological comorbidities in this study. Patients in the si-MALDI-TOF group had immunosuppressive medication more often than the ones in the control group (63.8\% versus $47.3 \%, P=0.066$ ). None of the patients had an HIV infection. The most common source of bacteremia was abdominal cavity (44.4\%), with second being urinary tract (16.9\%) (Table 1). Seven-day mortality was $2.4 \%(n=3)$ and 30-day mortality was $12.9 \%$ ( $n=16)$, with no statistically significant difference between study groups (data not shown).

\subsection{Microbiology and si-MALDI-TOF results}

Pseudomonas aeruginosa caused 21 (16.9\%) bacteremia episodes, Enterococcus spp. 62 (50\%), and AmpC-producing Enterobacteriaceae $41(33.1 \%)$. These results are shown in more detail in Table 2. Of all 103 monobacteremias, si-MALDI-TOF was done in 65 and yielded a result in 62 ( 3 bacteremias without a result: 1 E. faecium, 2 E. cloacae). Of polymicrobial bacteremias ( $n=21)$, si-MALDI-TOF was done in 14 and yielded a result in 7 (for the index bacteria).

\subsection{Early initiated empiric antibiotic treatment}

In 75.8\% ( $n=94$ ) of bacteremia episodes caused by the index bacteria, antibiotic treatment was either started or changed within $12 \mathrm{~h}$ after
Table 2

Number of bacteremic episodes according to the causative bacteria $(n=124)$.

\begin{tabular}{lllll}
\hline & $\begin{array}{l}\text { All } \\
\text { episodes }\end{array}$ & $\begin{array}{l}\text { Si-MALDI-TOF } \\
\text { group }\end{array}$ & $\begin{array}{l}\text { Control } \\
\text { group }\end{array}$ & $\begin{array}{l}P \\
\text { value }\end{array}$ \\
\hline $\begin{array}{l}\text { Pseudomonas } \\
\quad \text { aeruginosa }\end{array}$ & 21 & 14 & 7 & 0.265 \\
Enterococcus spp. & 62 & 33 & 29 & 0.588 \\
E. faecalis & 22 & 11 & 11 & \\
E. faecium & 37 & 21 & 16 & \\
E casseliflavum & 3 & 1 & 2 & \\
AmpC-producing & 41 & 22 & 19 & 0.754 \\
$\quad$ Enterobacteriaceae & & & & \\
E. aerogenes & 2 & 1 & 1 & \\
E. cloacae & 21 & 11 & 0 & \\
Serratia liquefaciens & 1 & 1 & 7 & \\
Serratia marcescens & 13 & 6 & 1 & \\
Morganella morganii & 4 & 3 & & \\
\hline
\end{tabular}

the positive blood culture draw; in $19.4 \%$ ( $n=24)$, no change was made to the ongoing inappropriate antibiotic treatment; and 6 patients received no antibiotic treatment at all. Subsequently, early initiated empiric antibiotic treatment was appropriate only in $22.6 \%(n=28)$ of the bacteremia episodes in this study, and in the remaining episodes, treatments were inappropriate.

\subsection{Impact of si-MALDI-TOF analysis on antibiotic treatment}

In 72.6\% ( $n=90)$ of all bacteremia episodes, patients received appropriate antibiotic treatment within $48 \mathrm{~h}$ from the first positive blood culture draw. In si-MALDI-TOF group, this percentage was 78.3\% $(n=54)$ and in control group $65.5 \%(n=36) ; P=0.112$ by univariate analysis (Table 3). A similar trend was seen in Kaplan-Meier survival analysis (Fig. 1). However, in BSIs caused by Enterococcus spp. ( $n=62)$, a significantly higher percentage of cases received appropriate antibiotic therapy within $48 \mathrm{~h}$ in si-MALDI-TOF group (87.9\% versus $65.5 \%$ in the control group, $P=0.036$ ). Results for different subgroups according to the index bacteria are shown in Table 3 .

A significant benefit of si-MALDI-TOF identification was seen in the treatment of bacteremic episodes in patients with immunosuppressive medication $(n=70)$. In si-MALDI-TOF group, $86.4 \%(n=38 / 44)$ and, in control group, $57.7 \%(n=15 / 26)$ cases had appropriate antibiotic treatment within $48 \mathrm{~h}$ from blood culture draw $(P=0.007)$, as shown in Fig. 2.

In general, median time from blood culture draw to receipt of appropriate antibiotic treatment was $33.5 \mathrm{~h}$ (interquartile range [IQR] 19.349.5). In si-MALDI-TOF group versus control group, median times were $31.9 \mathrm{~h}$ (IQR 14.9-46.8) and $39.7 \mathrm{~h}$ (IQR 21.2-61.4), respectively $(P=0.230)$.

\section{Discussion}

In this study, we showed that enhanced MALDI-TOF analysis, siMALDI-TOF, was valuable in guiding the antibiotic treatment of bacteremia episodes caused by Pseudomonas aeruginosa, Enterococcus spp., and AmpC-producing Enterobacteriaceae. Si-MALDI-TOF increased the proportion of appropriate antibiotic treatment within $48 \mathrm{~h}$ from the blood culture draw by $12.8 \%$. Significant improvement in proportion of patients with appropriate treatment was seen in Enterococcus spp. group (22.4\%, $P=0.036)$. Of all cases, the antibiotic treatment was appropriate only in $22.6 \%$ of cases within $12 \mathrm{~h}$ from the blood culture draw but was improved to $72.6 \%$ within $48 \mathrm{~h}$. In patients with immunosuppressive medication, si-MALDI-TOF significantly increased the percentage of cases receiving appropriate antibiotic treatment within $48 \mathrm{~h}$ from blood culture draw.

In a study by Vlek and colleagues, it was demonstrated that, in 218 patients with BSI, rapid MALDI-TOF was associated with an $11.3 \%$ increase in the proportion of appropriate antibiotic treatment after $24 \mathrm{~h}$ 
Table 3

Number of bacteremia episodes (BE) on appropriate antibiotic treatment within $48 \mathrm{~h}$ from the first positive blood culture draw.

\begin{tabular}{|c|c|c|c|c|c|}
\hline & & $\begin{array}{l}\text { Total number } \\
n(\%)\end{array}$ & Si-MALDI-TOF group & Control group & P value \\
\hline \multicolumn{2}{|c|}{ All bacteremia episodes in study group } & $90 / 124(72.6)$ & $54 / 69(78.3)$ & $36 / 55(65.5)$ & 0.112 \\
\hline \multirow[t]{3}{*}{ BE caused by } & Pseudomonas aeruginosa & $16 / 21(76.2)$ & $11 / 14(78.6)$ & $5 / 7(71.4)$ & $>0.99$ \\
\hline & Enterococcus spp. & $48 / 62(77.4)$ & $29 / 33(87.9)$ & $19 / 29(65.5)$ & 0.036 \\
\hline & Amp-C producing Enterobactariaceae & $26 / 41(63.4)$ & $14 / 22(63.6)$ & $12 / 19(63.2)$ & 0.975 \\
\hline
\end{tabular}

of blood culture positivity (64.0\% vs. $75.3 \%$ ) (Vlek et al., 2012). This result is in line with our study, although for Enterococcus spp., the impact of si-MALDI-TOF was greater ( $65.5 \%$ vs. $87.9 \%$ ), suggesting special benefit in that group.

In another study by Clerc et al. including 202 episodes of gramnegative bacteremias, enhanced MALDI-TOF analysis had an impact on empiric antibiotic therapy in $35.1 \%$ of bacteremia episodes and in $59.3 \%$ of the episodes caused by AmpC-producing Enterobacteriaceae ( $n=27$ ) (Clerc et al., 2013). Compared to our study, the number of bacteremias caused by the Enterobacter spp. and other AmpC-producing Enterobacteriaceae was lower. In spite of the higher number of BSIs caused by AmpC-producing Enterobacteriaceae, we could not detect any difference between the study groups. One possible explanation is that, in the study by Clerc et al., only patients for whom ID consultation was performed were included.

In a recent study, the benefit of rapid MALDI-TOF identification was evaluated in a setting with preexisting antimicrobial stewardship team program (Osthoff et al., 2016). Although it did not have an impact on the duration of intravenous antibiotic therapy, it led to lowered admission frequency to the intensive care unit after BSI onset. The MALDI-TOF analysis seemed to offer an extra benefit in a subgroup that included AmpC-producing Enterobacteriaceae and Enterococcus spp. In the 2 studies, additional benefit of MALDI-TOF method was suggested in treating patients with BSIs caused by the index bacteria in our study (Clerc et al., 2013; Osthoff et al., 2016).

In the recent study by Jeon et al., the use of MALDI-TOF alone did not significantly reduce the time to effective therapy (Jeon et al., 2018). This is in contrast to our study, which adds to the body of evidence on benefits of si-MALDI-TOF on antibiotic treatment of patients with BSIs caused by the index bacteria in our study. However, further studies will be needed, as noted in a recent meta-analysis, which also emphasized that earlier information should also lead to an appropriate clinical response (Dixon et al., 2015). This idea is further supported in a recent study on 239 BSI patients showing that MALDI-TOF method combined with antimicrobial stewardship intervention was more powerful than MALDI-TOF MS alone (Beganovic et al., 2017).

An interesting finding is the impact of si-MALDI-TOF in a subgroup of patients with immunosuppressive medication. In this group of patients, the proportion on appropriate antibiotic treatment within $48 \mathrm{~h}$ from the blood culture draw was significantly larger in the si-MALDI-TOF group than in the control group. This finding is difficult to explain. Looking only at bacteremic episodes in patients with immunosuppressive medication with inappropriate initial empiric antibiotic therapy, the difference remains ( $n=54,81.8 \%$ vs. $46.7 \%, P=0.008$ ). The proportions of different bacteria are also the same (e.g., Enterococcus spp. 52.9\%, rest of the data not shown). One possible explanation is that the treating

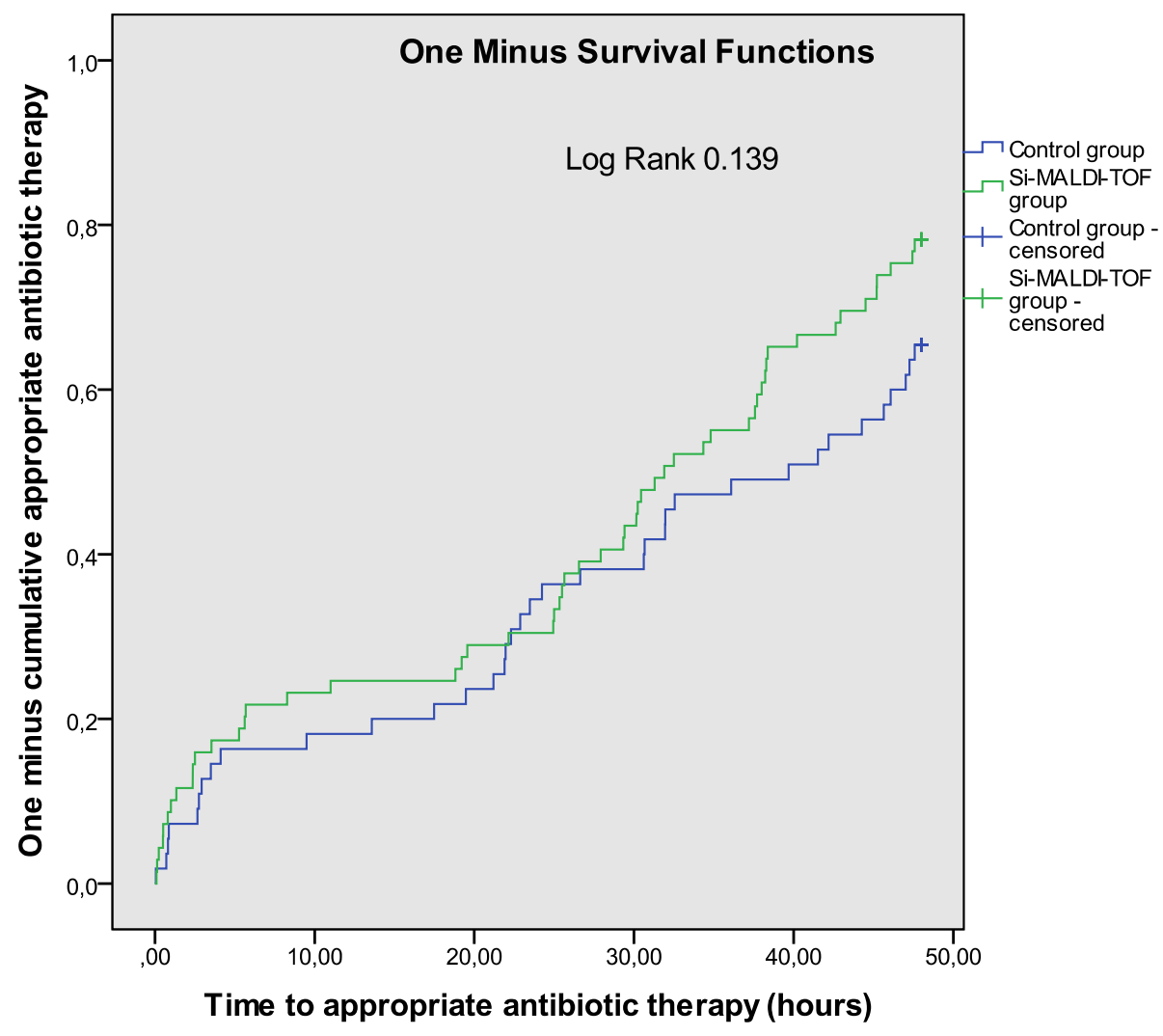

Fig. 1. Kaplan-Meier survival analysis (log rank) between the 2 study groups, censored at $48 \mathrm{~h}$. 


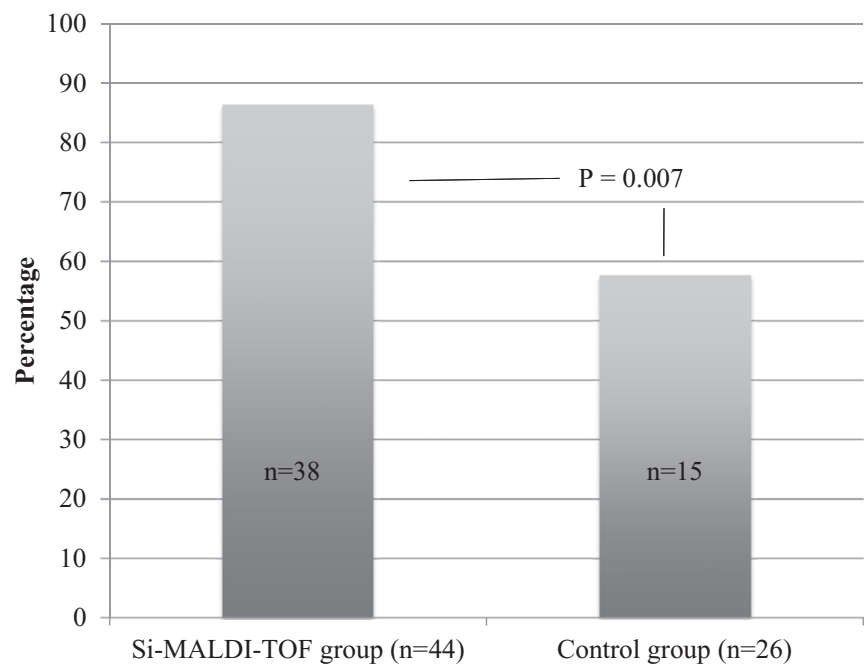

Fig. 2. Percentages of appropriate antibiotic treatment within $48 \mathrm{~h}$ in bacteremic episodes of patients with immunosuppressive medication $(n=70)$.

physicians observe this group of patients more vigilantly and take full advantage of the information provided by the rapid diagnostics.

In clinical work, the antibiotic treatment is usually initiated after blood culture draw; in this study in $75.8 \%$ of cases. However, the antibiotic treatment was appropriate only in $22.6 \%$ of bacteremic episodes within $12 \mathrm{~h}$ after the blood culture draw. Empiric antibiotic therapy is often not changed until further microbial or clinical evidence is obtained, which usually takes at least $48 \mathrm{~h}$. This highlights the importance of methods like si-MALDI-TOF that speed up the identification of bacteria in patients with BSI. Indeed, in clinical routine, information acquired from short-incubation MALDI-TOF method led to a change in empiric antibiotic treatment in $20 \%$ of patients with bacteremia (179 antibiotic prescriptions analyzed), and three quarters of these changes were considered rational by the authors (Kock et al., 2017).

The most plausible explanation why si-MALDI-TOF analysis brought a statistically significant benefit particularly in enterococcal bacteremias is the natural resistance profile of these microbes. Antibiotics effective also against enterococci are seldom included in empiric (in $\mathrm{HUH}$ often second- or third-generation cephalosporin) as well as in secondline antibiotic treatment (often a more broad spectrum beta-lactam). This is especially highlighted in BSI caused by E. faecium, which is often resistant to beta-lactams and requires vancomycin implemented in the regimen. Indeed, in $83.9 \%(n=52)$ of all enterococcal bacteremias, the early empiric therapy was inappropriate. An effective coverage was obtained by expanding the regimen with either vancomycin or linezolid $(67.3 \%, n=35)$ or, alternatively, with enterococcal betalactam $(32.7 \%, n=17)$. This was seen clearly in E. faecium bacteremias $(n=37)$ where vancomycin was needed in $86.5 \%(n=32)$ of cases in order to make the antibiotic therapy effective.

The strength of this study is its focus on the group of bacteria requiring special attention towards antibiotic therapy. Another strength is that the impact of si-MALDI-TOF information on antibiotic treatment could be evaluated independently of antimicrobial stewardship team since it was introduced to a routine clinical setting (i.e., real-world data). Although this is a single-center study, patient population was quite wide and diverse. Advantage of a single-center study is that all samples were analyzed in a single microbial university laboratory (HUSLAB).

There are several limitations in our study. It is a single-center study and is retrospective. Bacteremia episodes were divided into 2 study groups as described above, so presence of confounding factors is possible. If si-MALDI-TOF did not recognize the index bacteria from positive blood culture, clinicians did not receive any microbial information earlier, and hence, these bacteremia episodes were placed in the control group. Additionally, appropriate de-escalation of antibiotic therapy was not evaluated. Importantly, quite a high number of patients had neutropenia or were otherwise immunosuppressed, and in many cases, the source of infection was the abdominal cavity. These are situations where de-escalation is often not plausible even when bacteria in blood are recognized. In addition, the natural resistance profile of the bacteria in our study often necessitates the use of broad-spectrum antibiotics. In polymicrobial infections, the appropriateness of antibiotic therapy was assessed from the perspective of the index bacteria. However, in every polymicrobial bacteremia episode, appropriateness of antibiotic therapy was reached when index bacteria were covered. SiMALDI-TOF provides faster recognition to species level but no further information about antimicrobial susceptibility of the bacteria. Thus, our findings might not be generalizable to a setting where prevalence of more resistant bacteria (e.g., VRE, MDR Pseudomonas) is higher. The number of cases was too low to properly assess this method's impact on mortality. However, as noted in a recent meta-analysis, true mortality benefits may be difficult to detect when assessing the benefits of molecular rapid detection test, and time to appropriate antibiotic therapy is a better indicator (Timbrook et al., 2017).

\section{Conclusion}

Si-MALDI-TOF is a useful tool in the treatment of patients with bloodstream infections. It increases the number of patients receiving appropriate antibiotic treatment within $48 \mathrm{~h}$ from blood culture draw. A statistically significant impact was observed in patients with BSI caused by Enterococcus spp. and in a subgroup of patients with immunosuppressive medication.

\section{Funding}

This study has received financial support from Helsinki University Hospital (EVO grant).

\section{Declaration of interest}

Authors report no conflict of interest relevant to this study. MH has received conference invitation from Astellas and a speaker honorarium from MSD and Ratiopharm and has recent consultancies with Pfizer. AN has received a congress invitation from MSD. TM has received a speaker honorarium from CSL Behring and Octapharma; congress invitation from Pfizer, CSL Behring, and Gilead; and a grant from Sanguin. PK has received a speaker honorarium from MSD. VJA has received a speaker honorarium from Astellas, Roche, MSD, and Pfizer and has recent consultancies with MSD and Pfizer and study participation with Astellas and MSD.

\section{References}

Beganovic M, Costello M, Wieczorkiewicz SM. Effect of matrix-assisted laser desorption ionization-time of flight mass spectrometry (MALDI-TOF MS) alone versus MALDITOF MS combined with real-time antimicrobial stewardship interventions on time to optimal antimicrobial therapy in patients with positive blood cultures. J Clin Microbiol 2017;55:1437-45.

Bizzini A, Greub G. Matrix-assisted laser desorption ionization time-of-flight mass spectrometry, a revolution in clinical microbial identification. Clin Microbiol Infect 2010;16:1614-9.

Clerc O, Prod'hom G, Vogne C, Bizzini A, Calandra T, Greub G. Impact of matrix-assisted laser desorption ionization time-of-flight mass spectrometry on the clinical management of patients with gram-negative bacteremia: a prospective observational study. Clin Infect Dis 2013;56:1101-7.

Delport JA, Strikwerda A, Armstrong A, Schaus D, John M. MALDI-ToF short incubation identification from blood cultures is associated with reduced length of hospitalization and a decrease in bacteremia associated mortality. Eur J Clin Microbiol Infect Dis 2017;36:1181-6.

Dixon P, Davies P, Hollingworth W, Stoddart M, MacGowan A. A systematic review of matrix-assisted laser desorption/ionisation time-of-flight mass spectrometry compared to routine microbiological methods for the time taken to identify microbial organisms from positive blood cultures. Eur J Clin Microbiol Infect Dis 2015;34:863-76. 
ARTICLE IN PRESS

6

M. Halavaara et al. / Diagnostic Microbiology and Infectious Disease xxx (xxx) xxx

Drancourt M. Detection of microorganisms in blood specimens using matrix-assisted laser desorption ionization time-of-flight mass spectrometry: a review. Chin Microbiol Infect 2010;16:1620-5.

Huang AM, Newton D, Kunapuli A, Gandhi TN, Washer LL, Isip J, et al. Impact of rapid organism identification via matrix-assisted laser desorption/ionization time-of-flight combined with antimicrobial stewardship team intervention in adult patients with bacteremia and candidemia. Chin Infect Dis 2013;57:1237-45.

Ibrahim EH, Sherman G, Ward S, Fraser VJ, Kollef MH. The influence of inadequate antimicrobial treatment of bloodstream infections on patient outcomes in the ICU setting. Chest 2000;118:146-55.

Idelevich EA, Schule I, Grunastel B, Wullenweber J, Peters G, Becker K. Rapid identification of microorganisms from positive blood cultures by MALDI-TOF mass spectrometry subsequint to very short-term incubation on solid medium. Chin Microbiol Infect 2014;20: 1001-6.

Jon YD, Seong H, Kim D, Ahn MY, Jung IY, Jeong SJ, et al. Impact of matrix-assisted laser desorption/ionization time of flight mass spectrometric evaluation on the clinical outcomes of patients with bacteremia and fungemia in clinical settings lacking an antimicrobial stewardship program: a pre-post quasi experimental study. BMC Infect Dis 2018;18. [385-018-3299-y].

Kans CI, Kim SH, Kim HB, Park SW, Chou YJ, Oh MD, et al. Pseudomonas aeruginosa bacteremia: risk factors for mortality and influence of delayed receipt of effective antimicrobial therapy on clinical outcome. Chin Infect Dis 2003;37:745-51.

Kock R, Wullenweber J, Horn D, Lanckohr C, Becker K, Idelevich EA. Implementation of short incubation MALDI-TOF MS identification from positive blood cultures in routine diagnostics and effects on empiric antimicrobial therapy. Antimicrob Resist Infect Control 2017;6. [12-017-0173-4. eCollection 2017].

Kohlmann R, Hoffmann A, Gees G, Gatermann S. MALDI-TOF mass spectrometry following short incubation on a solid medium is a valuable tool for rapid pathogen identificatimon from positive blood cultures. Int J Med Microbiol 2015;305:469-79.

Leibovici L, Shraga I, Drucker M, Konigsberger H, Samara Z, Pitlik SD. The benefit of approprate empirical antibiotic treatment in patients with bloodstream infection. J Intern Med 1998;244:379-86.
Lodise TP, Jr Patel N, Kwa A, Graves J, Furuno JP, Graffunder E, et al. Predictors of 30-day mortality among patients with Pseudomonas aeruginosa bloodstream infections: inpact of delayed appropriate antibiotic selection. Antimicrob Agents Chemother 2007; 51:3510-5.

Martiny D, Debaugnies F, Gateff D, Gerard M, Aoun M, Martin C, et al. Impact of rapid microbial identification directly from positive blood cultures using matrix-assisted laser desorption/ionization time-of-flight mass spectrometry on patient management. Chin Microbial Infect 2013;19:E568-81.

Osthoff M, Gurtler N, Bassetti S, Balestra G, March S, Pargger H, et al. Impact of MALDI-TOF mass spectrometry based identification directly from positive blood cultures on patent management: a controlled clinical trial. Chin Microbiol Infect 2017;23(2):78-85.

Patel TS, Kaakeh R, Navel JL, Newton DW, Stevenson JG. Cost analysis of implementing matrix-assisted laser desorption ionization-time of flight mass spectrometry plus real-time antimicrobial stewardship intervention for bloodstream infections. J Chin Microbial 2016;55:60-7.

Perez KK, Olsen RJ, Musick WL, Cernoch PL, Davis JR, Land GA, et al. Integrating rapid pathogen identification and antimicrobial stewardship significantly decreases hospital costs. Arch Pathol Lab Med 2013;137:1247-54.

Seifert $\mathrm{H}$. The clinical importance of microbiological findings in the diagnosis and management of bloodstream infections. Chin Infect Dis 2009;48(Suppl.4): S238-45.

Skogberg K, Lyytikainen O, Ollgren J, Nuorti JP, Ruutu P. Population-based burden of bloodstream infections in Finland. Chin Microbiol Infect 2012;18:E170-6.

Timbrook TT, Morton JB, McConeghy KW, Caffrey AR, Mylonakis E, LaPlante KL. The effect of molecular rapid diagnostic testing on clinical outcomes in bloodstream infections: a systematic review and meta-analysis. Chin Infect Dis 2017;64:15-23.

Vlek AL, Bonten MJ, Bel CH. Direct matrix-assisted laser desorption ionization time-offlight mass spectrometry improves appropriateness of antibiotic treatment of batteremil. PLo One 2012;7, e32589.

Zasowski EJ, Clays KC, Lagnf AM, Davis SL, Rybak MJ. Time is of the essence: the impact of delayed antibiotic therapy on patient outcomes in hospital-onset Enterococcal bloodstream infections. Chin Infect Dis 2016;62:1242-50.

Please cite this article as: M. Halavaara, A. Nevalainen, T. Martelius, et al., Impact of short-incubation MALDI-TOF MS on empiric antibiotic therapy

in bloodstream infections caused by ..., Diagnostic Microbiology and Infectious Disease, https://doi.org/10.1016/j.diagmicrobio.2018.11.009 